\title{
Solar based 3D Printed Protective Mask Imprinted with Extracted Oil and UV Light to Kill Corona Virus
}

\section{Sreedevi Paramparambath, Mohammed Houkan, Shady Galal Hussein Haggagy, Kishor Kumar Sadasivuni` \\ Centre for Advanced Materials, Qatar University, P.O. Box 2713, Doha, Qatar *Corresponding author: kishorkumars@qu.edu.qa}

\section{AIM}

- Fabrication of 3D printed reusable user-friendly protective mask that protects from COVID-19 virus by trapping the virus and killing it using UV light.

- Found new technology to trap and kill COVID-19 virus using oil trapping and UV light, respectively.

- Benefits of using this mask is, that along with disinfecting the inhaled air it can remove the suffocation problem also.

- Reduces the amount of wastes caused by mask disposal.

- Solar sources used to supply the power to the proposed device.

\section{INTRODUCTION}

- In this current scenario of global pandemic we are fighting against COVID in different ways.

- Coronavirus disease is an infectious disease caused by a newly discovered virus called corona. It is transmitted from the droplets formed when infected people sneezes, coughs or exhales.

- Currently 30,697,825+ people are globally affected by this virus.

\section{Disadvantages of commercially available masks:}

- The purpose of commercially available mask is to keep respiratory droplets from reaching others to aid person from virus spread.

- However mask with one-way valves or vent allows air to be exhaled through a hole, which can result in expelled respiratory droplets that can reach others.

- Those people who wears commercially available mask if touches nose, eyes, mouth after removing mask without washing hands can still catch the virus infection.

- Respiratory/suffocation problems due to the usage of this masks

- Causes a large amount of waste due to the usage of disposable mask.

- The improper disposal of this commercially available mask leads to the spreading of virus to humans as well as animals.

\section{Advantages of developed 3D Printed masks:}

- The fabrication of this protective mask which kills the virus instantly is very effective and this can be designed using 3D printing according to the facial features of individuals.

- 3D Printing is a fabrication methodology used for printing 3D objects based on controlled layer deposition of printable material until final structure is obtained and so it is known as additive manufacturing.

- It is often termed as rapid prototyping.

- In this 3D printed mask we can imprint naturally extracted oil and UV light to kill the virus.

- This mask provides complete protection from virus rather than commercially available mask which act as a barrier and provides partial protection.

\section{CONCLUSION}

- 3D printed protective mask can be designed according to each individual facial features which helps in complete prevention of virus.

- This protective mask can kill virus rather than acting like a barrier. This mask can be used by people suffering from breathing problems which is an added advantage over commercially available masks.

- Using commercially available masks have great risk of spreading, if one touches the mask while using it. And the masks with one-way valves or vents allows the expelled respiratory droplets to reach others and facilitates spreading of virus.

- 3D printed mask can be used over the commercially available mask as it is user friendly and give complete protection from virus and its spread.

- By using 3D printed reusable mask we can reduce the wastes caused by commercially available disposable masks.

- The 3D printed mask will be connected to solar hat to generate the power for the device.

\section{REFERENCES}

O’Dowd, K., Nair, K.M., Forouzandeh, P., Mathew, S., Grant, J., Moran, R., Bartlett, J., Bird, J. and Pillai, S.C., 2020. Face Masks and Respirators in the Fight against the COVID-19 Pandemic: A Review of Current Materials, Advances and Future Perspectives. Materials, 13(15), p.3363. Belhouideg, S., 2020. Impact of 3D printed medical equipment on the management of the Covid19 pandemic. The International Journal of Health Planning and Management

\section{ACKNOWLEDGEMENT}

We are thankful to Center for Advanced Materials (CAM) and Central Laboratory Unit (CLU) Qatar University for continuous support for this work. This work was supported by the RRC-2-063

The statements made herein are solely the responsibility of the authors. 